\title{
A compact fractal-based asymmetrical dipole antenna for RFID tag applications
}

\begin{abstract}
Size reduction, among many other parameters, is one of the main challenges encountering antenna design for RFID tag applications. For this, different fractal geometries are found to be a good candidate. In this paper, a design of compact fractal-based asymmetrical dipole antenna integrated with Split Ring Resonator (SRR) is proposed. A Minkowski fractal tag loaded with a square SRR on its backplane, is designed for Ultra High Frequency (UHF) band Radio Frequency Identification (RFID). Modeling and performance assessment of the proposed antenna are carried out using CST microwave studio. Results reveal that the antenna has a size of $82 \times 87 \times 1.6 \mathrm{~mm} 3$ and provides a read range of $2.17 \mathrm{~m}$. These parameters together with other radiation characteristic make it suitable for RFID applications.
\end{abstract}

Keyword: RFID; Tag antenna; Compact size antenna; Fractal antenna, SRR 